and serosorting, we examined how often men have UAI and report serosorting with three casual partner types that differ in the degree of familiarity.

Methods We included 240 HIV-negative men of the Amsterdam Cohort Study among MSM. We distinguished three casual partner types-one-night stand ("met by chance and had sex only once"); multiple-time casual partner ("met by chance and had sex several times"); and sex buddy ("contacted on a regular basis for sex but not considered a steady partner"). Serosorting was defined as UAI with an HIV-negative partner. GEE analyses were performed to examine the association between casual partner type and sexual risk behaviour.

Results 240 participants accounted for 362 reports of anal intercourse, of which 206 (57\%) with one-night stands, 95 (26\%) with multiple-time casual partners, and $61(17 \%)$ with sex buddies. The proportion of UAI was $19 \%$ (39/206) with one-night stands, $20 \%$ (19/95) with multiple-time casual partners, and 34\% (21/61) with sex buddies. When performing UAI, 21\% (8/39) reported serosorting with one-night stands, $42 \%(8 / 19)$ with multiple-time casual partners, and $52 \%(11 / 21)$ with sex buddies. Analyses revealed that only men with a sex buddy were significantly more likely to have UAI (OR [95\% CI] 2.39 [1.39 to 4.09]), but were also more likely to practice serosorting than men with a one-night stand (OR $[95 \% \mathrm{CI}]$ 5.20 [1.20 to 22.52]). No differences were found between the multiple-time casual partner and the other partner types.

Conclusions Men with a sex buddy had more UAI but also reported more serosorting than men with a one-night stand. As a result, the proportion of UAI without serosorting is lower for men with a sex buddy, and therefore men might have less UAI at risk for HIV with this partner type. However, the quality of serosorting with casual partners and hence their protective value against HIV needs to be further established. At this time, we suggest that a distinction between the one-night stand and the sex buddy should be incorporated in future studies as risk behaviour of MSM is significantly different between these partner types.

\section{P1-S2.48 EXPONENTIAL GROWTH OF LYMPHOGRANULOMA VENEREUM DIAGNOSES IN THE UK: INVESTIGATION OF THE LARGEST DOCUMENTED OUTBREAK AMONG MEN WHO HAVE SEX WITH MEN}

doi:10.1136/sextrans-2011-050108.105

G Hughes, S Alexander, I Simms, S Conti, C Powers, C Ison. Health Protection Agency, London, UK

Background With over 1500 cases reported, the UK now has the largest documented outbreak of Lymphogranuloma venereum (LGV) among men who have sex with men (MSM). Of particular concern is the recent exponential rise in cases - a third of all cases have been reported since 2010. We investigated the characteristics of this outbreak and specifically whether any factors were associated with the sharp upsurge in cases in 2010.

Methods The UK LGV Incident Group launched a diagnostic reference service and enhanced surveillance of LGV in the UK in October 2004. All symptomatic patients attending UK GUM clinics who were chlamydia-positive or contacts of positive cases were referred to STBRL in London and later also to the Scottish Bacterial Sexually Transmitted Infections Reference Laboratory in Edinburgh for confirmation. Enhanced LGV surveillance forms were completed by clinicians caring for cases and submitted to the HPA for analysis. An epidemic curve was plotted and the point of exponential growth estimated. The characteristics of cases prior to and during the exponential growth phase were compared.

Results Since 2003, 1570 LGV cases have been reported in the UK, of which 1268 (81\%) had enhanced surveillance forms available. All but 8 (99\%) were in MSM and most (77\%) were seen in London, Brighton and Manchester. The point of exponential growth was estimated as 1st October 2009. Compared to cases prior to this date, cases in the growth phase were more likely to be older (41\% vs $38 \%$ aged over 40 ), to be HIV positive ( $84 \%$ vs $78 \%$ ), to be HCV antibody positive ( $27 \%$ vs $22 \%$ ), to report unprotected anal intercourse (UAI) ( $80 \%$ vs $77 \%$ ) and fisting (19\% vs $14 \%$ ), to present outside London ( $32 \%$ vs $27 \%$ ), and to have acquired LGV in the UK (91\% vs $87 \%$ ). Overall, median case age was 38, $84 \%$ were white and $11 \%$ reported they acquired the infection outside the UK, mostly in Western European countries associated with the epidemic. UAI was reported by $78 \%$ of cases and at least 50 were identified as re-infections.

Conclusions LGV is becoming endemic in the UK and is characterised by HIV positive MSM involved in dense sexual networks. Although cases associated with the recent upsurge are more geographically widespread, there is no evidence of dispersal among lower risk or HIV negative MSM. LGV control will require intensified awareness raising and outreach among at risk populations. Regression modelling of key variables is underway to investigate and validate these findings.

\section{P1-S2.49 CHLAMYDIA AND GONORRHOEA TRANSMISSION AMONG MEN WHO HAVE SEX WITH MEN IN AMSTERDAM, IS INDEPENDENT OF NETWORK FACTORS OR PARTNERSHIP CHARACTERISTICS}

doi:10.1136/sextrans-2011-050108.106

${ }^{1} \mathrm{~A}$ Matser, ${ }^{2} \mathrm{~T}$ Heijman, ${ }^{1} \mathrm{R}$ Geskus, ${ }^{1} \mathrm{H}$ de Vries, ${ }^{1} \mathrm{H}$ Fennema, ${ }^{1} \mathrm{M} \mathrm{S}$ van der Loeff. ${ }^{1}$ Public Health Service, Amsterdam, Netherlands; ${ }^{2}$ Cluster of Infectious diseases, Department of research, Amsterdam, Netherlands

Background Partnerships are essential for transmission of sexually transmitted infections (STI). Among MSM, variation in partnerships is common. We have analysed risk factors for STI by studying partnerships.

Methods The study population was recruited from the STI outpatient clinic of the public health service of Amsterdam, the Netherlands. Inclusion criteria were male gender, having had sexual contact with men in the past 6 months, age $\geq 18$ years, and understanding of written Dutch or English. Recruitment occurred from July 2008 to August 2009. Participants were screened for chlamydia (CT), gonorrhoea (NG), syphilis, and HIV (opting-out strategy). Participants completed a questionnaire including demographics and detailed questions about sexual behaviour in self-defined relationships with a steady partner and the last three other partners within the previous 6 months. Logistic regression analysis was used to identify risk factors for CT and NG.

Results 2731 MSM reporting 7397 partnerships were included; median age was 39 years (IOR 31-45). CT prevalence was $12.5 \%$ $(n=342)$, NG prevalence was $11.1 \%(n=303)$, and CT/NG coinfection was found in $2.6 \%(n=71)$ of the MSM. Meeting partners online was common (53\% met at least one of the partners online), but not associated with STI (CT-OR 1.2 95\% CI 0.95 to 1.5 and NG-OR $1.295 \%$ CI 0.9 to 1.5), just as having concurrent partnerships (CT-OR 3.2 95\% CI 0.4 to 23.6 and NG-OR $1.395 \% \mathrm{CI}$ 0.3 to 5.6). In multivariable analysis, independent predictors of CT were having $=5$ known partners in the last 6 months (OR $1.795 \%$ CI 1.2 to 2.3), receptive unprotected anal intercourse (OR $2.195 \%$ CI 1.5 to 2.8 ), NG coinfection (OR $2.095 \%$ CI 1.5 to 2.8), and HIV coinfection (OR $1.895 \%$ CI 1.4 to 2.4). Independent predictors of NG were group sex (OR $1.595 \%$ CI 1.1 to 2.1), being in a different 10 -year age category than the partner (OR $1.495 \%$ CI 1.04 to 1.8), CT coinfection (OR $2.195 \%$ CI 1.5 to 2.8), and HIV coinfection (2.2 $95 \%$ CI 1.6 to 2.9 ). Age $=45$ years (OR $0.495 \%$ CI 0.2 to 0.6 ) and being bear type (OR $0.495 \%$ CI 0.2 to 0.9 ) were associated with lower NG risk. 